\title{
Las bibliotecas públicas también son para los mayores: resultados de una encuesta realizada a profesionales bibliotecarios españoles
}

\author{
Fco. Javier GARCÍA GÓMEZ \\ Universidad de Murcia. Facultad de Comunicación y Documentación \\ fjgg3@um.es
}

Recibido: Febrero 2015

Aceptado: Abril 2015

\begin{abstract}
Resumen: El colectivo de personas mayores es uno de los sectores de población cuya presencia en la sociedad española irá en aumento en los próximos años. Las bibliotecas públicas, como centro de servicios y recursos de interés para su comunidad, deben considerar a este colectivo como un destinatario preferente a la hora de planificar, diseñar y ofrecer su oferta de servicios. Presentamos los resultados de una encuesta realizada a varias bibliotecas públicas españolas para averiguar qué servicios y recursos de interés para el colectivo de personas mayores ofrecen, los usos más comunes, y las tendencias que se pueden prever a corto y medio plazo
\end{abstract}

Palabras clave: Bibliotecas Públicas; España; Personas Mayores; Recursos Bibliotecarios; Servicios Bibliotecarios.

\section{Public libraries are also for adults: results of a survey of professional librarians in Spanish}

\begin{abstract}
The group of seniors is one of the sectors of the population whose presence in the Spanish society will increase in the coming years. Public libraries, as a center of services and resources of interest to the community, should consider this group as a preferred destination for the planning, design and deliver their service offerings. We present the results of a survey of Spanish public libraries to find out what services and resources of interest to the group of elderly service, the most common uses, and trends that can be expected in the short and medium term
\end{abstract}

Keywords: Library Resources; Library Services; Older People; Public Libraries; Spain.

\section{INTRODUCCIÓN}

La sociedad española está experimentando cambios singulares en la última década, especialmente desde el punto de vista demográfico y de estructura de edades. De hecho, se reconoce que la sociedad española tiende a un claro 
envejecimiento en cuanto a que el porcentaje de personas mayores ${ }^{1}$ de 55 años crece de manera sobresaliente mientras que los tramos de edad más jóvenes tienden a estancarse o a disminuir (Pérez Díaz, 2010). Las razones de este gran cambio demográfico son, por una parte, la disminución precisamente de los niveles de natalidad y, por otra, la mayor expectativa de vida de los mayores españoles debido fundamentalmente a los avances médicos, la mejora de las condiciones de vida y de las prestaciones sociales en su conjunto.

Por otro lado, el colectivo de personas mayores no es reconocido en el ámbito bibliotecario español como usuarios habituales de los servicios de las bibliotecas públicas españolas ${ }^{2}$. Tradicionalmente, el uso que han hecho de los servicios bibliotecarios ha sido de manera muy esporádica, para fines muy concretos, y con unas tasas de participación muy bajas (García y Díaz, 2001). No obstante, en los últimos años, en el seno del conjunto de bibliotecas públicas españolas se ha reconocido la necesidad de atender convenientemente también las necesidades e intereses de este grupo social (Ares Seijo, 2004; Saloni, 2008) y muchas de las bibliotecas públicas de nuestra geografía han adoptado un papel más activo en este sentido (García Gómez, 2008). No obstante lo dicho, este tema de investigación se encuentra poco documentado en la literatura profesional española y precisa de más estudios en este sentido (García Gómez, 2014).

Junto a esta realidad - la necesidad de atraer a este colectivo de usuarios a las bibliotecas, de proponer métodos de trabajo para conseguirlo y de exponer

1 Somos conscientes que el término "personas mayores” u otros como "tercera edad", "personas de edad avanzada”, “jubilados”, “ancianos”, o similares no resultan apropiados ni son los que mejor se ajustan para denominar a este colectivo de personas. Consideramos que la idea de tramos de edad o la propia categoría "mayores” no parecen del todo acertadas pues no hay que confundir necesidad con edad. De hecho, en la literatura científica del área de la geriatría o la sociología, no existe un consenso reconocido a la hora de denominar a aquellas personas que alcanzan o superan los 55, 60 o 65 años de edad, porque esta es otra cuestión en la que tampoco hay un acuerdo definitivo, es decir, a partir de qué edad podemos considerar a un ciudadano como "persona mayor". Consideramos que, de los términos existentes para referirse a este colectivo, "personas mayores" es el que tiene quizás una menor connotación negativa frente a otros como "viejos” o "ancianos” y que, sin ser el término más adecuado, hemos considerado su uso por encontrarse ampliamente presente entre la bibliografía científica relacionada. No obstante, insistimos en la opinión de que no es el más adecuado ni el definitivo y, siguiendo a Saloni (2008), haremos uso del mismo en cursiva

${ }^{2}$ En el estudio Opiniones y actitudes de los usuarios de las Bibliotecas Públicas del Estado, realizado en 2.009 a instancia de la Subdirección General de Coordinación Bibliotecaria del Ministerio de Cultura y de la Fundación Germán Sánchez Ruipérez, se aportan dos interesantes datos al respecto: por un lado, "la escasa incidencia que tienen las bibliotecas entre la población femenina a partir de los 45 años y, sobre todo, entre las mujeres de más edad” (p. 21); por otro lado, "Los [usuarios] menos numerosos, que suman un 5,2\%, son los mayores de 55 años; un $3,1 \%$ corresponde a adultos que tienen entre 55 y 64 años y un 2,1\%, personas de 65 o más años”. Documento disponible en http://travesia.mcu.es/portalnb/jspui/handle/10421/1621 
modelos de buena práctica en este sentido- no hay que perder de vista otra como es la situación actual de restricciones presupuestarias para la prestación de todo tipo de servicios públicos, que pone en entredicho la viabilidad del actual Estado del bienestar (Uroz, 2010) y las repercusión que puede tener tanto sobre la prestación y desarrollo de los servicios bibliotecarios, como sobre la calidad de vida y la propia longevidad de los considerados mayores.

Así las cosas, para tener un conocimiento más exacto del estado de la cuestión sobre los usos actuales que hacen los mayores de las bibliotecas públicas españolas, de la oferta de servicios orientados hacia este colectivo, y las tendencias de trabajo que se proyectan a corto y medio plazo, así como de los posibles efectos que sobre la prestación de estos servicios bibliotecarios está teniendo la actual situación de recortes presupuestarios, hemos elaborado una encuesta que hemos hecho llegar a profesionales bibliotecarios para recabar la información necesaria que nos ayudara a ofrecer algo más de luz sobre este asunto. El propósito de este artículo es precisamente mostrar los resultados de esta encuesta, analizar los datos, extraer las conclusiones derivadas del análisis, y exponer las tendencias de servicio que se deducen al respecto.

\section{OBJETIVOS Y METODOLOGÍA}

El objetivo principal de esta encuesta es averiguar qué usos hacen el colectivo de personas mayores de las bibliotecas públicas españolas, qué servicios y recursos les ofrecen éstas, qué tendencias de uso y servicio se derivan del análisis de los datos recopilados, y ofrecer propuestas de trabajo que puedan servir de guía de ruta a seguir por los profesionales bibliotecarios a la hora de gestionar proyectos de trabajo con este colectivo en mente. Otro de nuestros objetivos ha sido, de manera indirecta, el de concienciar y sensibilizar a la comunidad bibliotecaria española con las necesidades e intereses de las personas mayores y la necesidad de considerarlos como destinatarios de sus programas de actividades y servicios y que dejen de ocupar un lugar destacado entre los grandes olvidados de los proyectos bibliotecarios.

El método de trabajo seguido fue el siguiente: en primer lugar, se confeccionó la encuesta a partir de la aplicación que proporciona la Universidad de Murcia al personal docente e investigador de la misma (http://encuestas.um.es). Una vez construida la encuesta, nos dispusimos a darla a conocer a la comunidad bibliotecaria española. Para ello, a finales del mes de julio de 2.014, en primer lugar hicimos un llamamiento a la colaboración de los profesionales bibliotecarios españoles a través de la listas de distribución Iwetel y Públicas; este mismo llamamiento se repitió en estas listas de distribución, a modo de recordatorio, a finales del mes de septiembre de 2.014. Junto a Iwetel y Públicas, le dimos difusión a través de la lista de distribución BiblioMun, de la Red de Bibliotecas Públicas de la Región de Murcia, comunidad autónoma en la que residimos y en la 
que se ubica la Universidad de Murcia. Las fechas de envío de la información a BiblioMun fueron las mismas que Iwetel y Públicas.

Junto a la convocatoria realizada a través de las listas de distribución, aprovechamos también el potencial de captación y de alcance que tienen actualmente las redes sociales (García Giménez, 2010; Ovalle y Guardado, 2013), y utilizamos tanto FaceBook como Linkedin como medio de comunicación de la encuesta. Al igual que sucedió con las listas de distribución, hicimos un primer llamamiento por las redes sociales y, posteriormente, un recordatorio, en las mismas fechas que hicimos con Iwetel, Públicas y BiblioMun.

En el caso de FaceBook, la difusión de esta encuesta se ha hecho de tres modos: en primer lugar, a través de nuestro perfil personal en esta red social www.facebook.com/fjaviergarciagomez; posteriormente, creamos un "evento" https://www.facebook.com/events/527362637392003/ con la opción que facilita Facebook con la idea de tanto de publicitar la encuesta, como para dar a conocer experiencias de trabajo bibliotecario con mayores de las que íbamos teniendo noticia. Finalmente, decidimos "trasladarnos" a modo de FanPage con la categoría de "Comunidad" www.facebook.com/pages/Servicios-y-Recursos-para-PersonasMayores-en-Bibliotecas-P\%C3\%BAblicas/1523228797920825 porque ofrece más posibilidades de difusión de información, porque permite llegar a las bibliotecas públicas con presencia en esta red social, y porque asegura el seguimiento, de una manera más directa, del trabajo bibliotecario que van realizando y que pudiera estar relacionado con el colectivo de las personas mayores. Más concretamente, lanzamos varios mensajes dando a conocer los objetivos de la página y, al tiempo, fuimos visitando las páginas de Facebook de las bibliotecas que cuentan con esta modalidad de presencia en la red social, fuimos marcando la opción "Me gusta”, y a partir de aquí se fue generando una retroalimentación e interacción muchas veces directa, otras indirecta, que facilita el conocimiento de las actividades y servicios que ofrece cada biblioteca y, en consecuencia, de los que pudiera haber orientados hacia el sector de población de los mayores.

Una tercera vía de difusión de la encuesta fue contactando por email con las principales asociaciones profesionales de bibliotecarios del país ${ }^{3}$ con la intención no solo de informarles de la encuesta, sino también de aprovechar sus posibilidades de contacto con cada uno de los profesionales bibliotecarios

${ }^{3}$ Las asociaciones contactadas por email fueron: Asociación Andaluza de Bibliotecarios (AAB); ASNABI de Navarra; ANABAD y sus Unidades Territoriales de Aragón, Murcia, La Rioja, Galicia y Castilla-La Mancha, y Cantabria; Index de Murcia; ABIGRANCA de Gran Canaria; los Colegios Oficiales de Bibliotecarios y Documentalistas tanto de Cataluña como de Valencia; ABADIB de Baleares; ABITO de Toledo; ALDEE de Euskadi; y SEDIC. Una relación con las principales asociaciones profesionales españolas del mundo de las bibliotecas y áreas afines puede encontrarse en http://www.recbib.es/book/asociaciones-nacionales 
adscritos a las mismas. Las fechas de contacto fueron las mismas que en los casos de las lista de distribución.

Figura 1. FanPage de FaceBook Servicios y Recursos para Personas Mayores en Bibliotecas Públicas

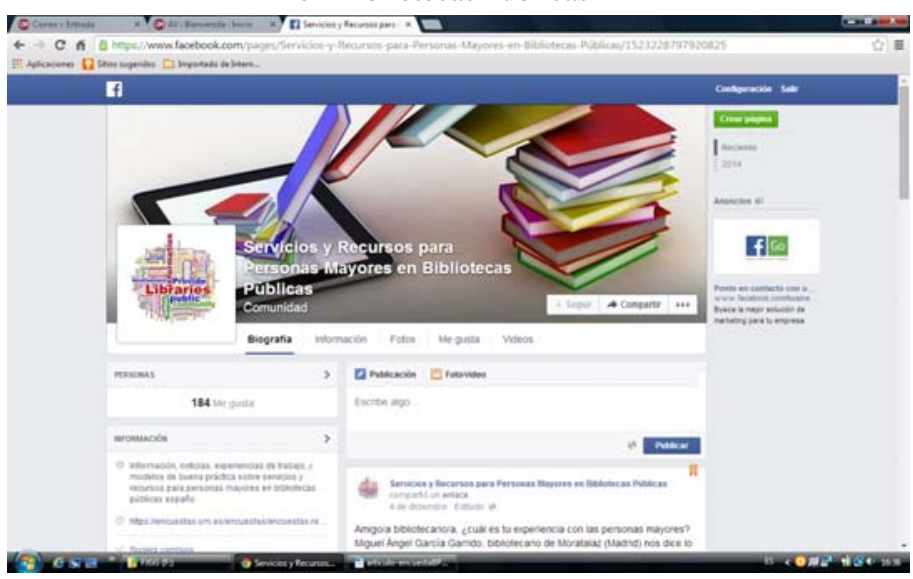

También hicimos mención a la existencia de la encuesta en nuestra comunicación presentada en el VII Congreso Nacional de Bibliotecas Públicas celebrado en Badajoz los días 12, 13, y 14 de noviembre de 2.014, animando a los profesionales bibliotecarios a participar en la misma (García Gómez, 2014).

La encuesta se encuentra disponible en la dirección https://encuestas.um.es/encuestas/encuestas.realizacion.insertar.gen?a=A572DF6E 28F42DBF4590A19C31BB4006

Figura 2. Encuesta online Servicios y Recursos para Personas Mayores en Bibliotecas Públicas

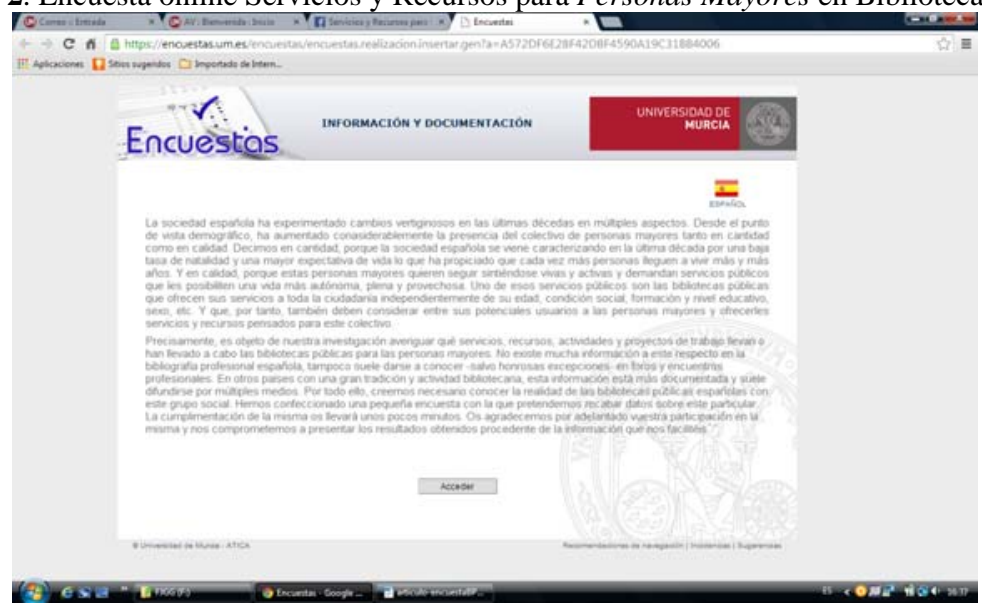




\section{LA ENCUESTA}

En primer lugar, hay que mencionar que cuando solicitamos a los profesionales bibliotecarios españoles su participación en la encuesta, nos comprometimos a adelantar un avance de resultados, el cual, utilizamos como recurso complementario para intentar asegurarnos su colaboración cumplimentando la encuesta. Este avance de resultados se presentó vía Iwetel, Públicas y BiblioMun el día 20 de noviembre de 2.014. En este avance de resultados adelantábamos aspectos parciales de los resultados que íbamos obteniendo del análisis de las encuestas cumplimentadas y que en este artículo procedemos a depurar con más detalle.

Mientras iniciábamos la redacción de este texto, algunas bibliotecas nos informaban que en breve - concretamente, a partir de enero de 2.015- tenían previsto poner en marcha alguna actividad o servicio con el colectivo de mayores como destinatarios del mismo, y que era su intención el participar en la encuesta una vez fueran extrayendo datos de interés para darlos a conocer en la encuesta. De ahí, que anunciáramos en las redes sociales que se ampliaba el plazo de recepción de encuestas hasta el mes de marzo de $2.015^{4}$.

La encuesta -que se acompaña como anexo al final de este texto- consta de 21 ítems que incorpora preguntas abiertas y cerradas, de forma que damos opción a que el profesional que cumplimenta la encuesta pueda aportar información complementaria, comentarios de interés, y cualquier otro dato que pueda ser relevante para los objetivos de nuestro estudio. Cada encuesta recaba, al principio, datos sobre la biblioteca participante, persona que responde la encuesta, cargo que ocupa en la institución bibliotecaria que participa en la encuesta, y fecha de cumplimentación de la misma. A continuación, se entra en el meollo de la cuestión y se plantea, a quien cumplimenta la encuesta, las preguntas que aparecen encuadradas en bloques de información: uso de la biblioteca; espacios y áreas físicas de la biblioteca; secciones y colecciones bibliotecarias; sede web de la biblioteca; servicios y actividades; y un apartado final de observaciones y comentarios de interés.

${ }^{4}$ Véase la noticia anunciada en la página de Facebook Servicios y Recursos para Persdonas Mayores en Bibliotecas Públicas www.facebook.com/1523228797920825/photos/ pb.1523228797920825.-2207520000.1426591301./1550602251850146/?type=1\&theater 
Tabla 1. Categorías temáticas de la encuesta y descripción de cada bloque temático

\begin{tabular}{|c|c|}
\hline CATEGORÍAS & DESCRIPCIÓN Y FINALIDAD \\
\hline $\begin{array}{l}\text { IDENTIFICACIÓN } \\
\text { DE LA BIBLIOTECA }\end{array}$ & $\begin{array}{l}\text { Se recaba información sobre la biblioteca participante, la persona } \\
\text { que rellena la encuesta, su cargo en la biblioteca, y la fecha de } \\
\text { cumplimentación }\end{array}$ \\
\hline $\begin{array}{l}\text { USO DE LA } \\
\text { BIBLIOTECA }\end{array}$ & $\begin{array}{l}\text { Se recoge información de uso o no uso de la biblioteca por parte } \\
\text { de los mayores, y qué usos hacen de la biblioteca de forma más } \\
\text { usual }\end{array}$ \\
\hline $\begin{array}{l}\text { ESPACIOS Y ÁREAS } \\
\text { FÍSICAS }\end{array}$ & $\begin{array}{l}\text { Se averigua si la biblioteca cuenta con algún área o espacio físico } \\
\text { pensado exclusivamente para este colectivo, además de las } \\
\text { condiciones de accesibilidad física que, en líneas generales, } \\
\text { ofrece la biblioteca }\end{array}$ \\
\hline $\begin{array}{l}\text { SECCIONES Y } \\
\text { COLECCIONES }\end{array}$ & $\begin{array}{l}\text { Se consulta si la biblioteca presenta alguna sección o colección } \\
\text { de documentos específica, independientemente de su soporte o } \\
\text { formato, orientada hacia los mayores }\end{array}$ \\
\hline SEDE WEB & $\begin{array}{l}\text { Se pregunta por la existencia o no de un apartado o sección } \\
\text { específica en la sede web de la biblioteca (en el caso de que } \\
\text { cuente con sucursal virtual), y el tipo de información o servicio } \\
\text { que se suministra }\end{array}$ \\
\hline $\begin{array}{l}\text { SERVICIOS Y } \\
\text { ACTIVIDADES }\end{array}$ & $\begin{array}{l}\text { Se indaga acerca del servicio, recursos, actividad o proyecto de } \\
\text { trabajo concreto sobre el que la biblioteca da a conocer, si el } \\
\text { mismo está en marcha o ya ha finalizado, si es permanente en el } \\
\text { tiempo u ocasional, y en el que caso de que no ofrezcan nada al } \\
\text { respecto si existe la previsión de diseñar u ofrecer a corto o } \\
\text { medio plazo algún servicio o actividad específica para y/o con } \\
\text { los mayores así como su duración temporal. Asimismo, se } \\
\text { interroga a las bibliotecas que han respondido positivamente, por } \\
\text { las características de la acción bibliotecaria indicada, por los } \\
\text { objetivos perseguidos (tanto generales como específicos), por los } \\
\text { destinatarios (si son personas mayores en general, o si es para un } \\
\text { tramo de edad específico -por ejemplo, para mayores con edades } \\
\text { comprendidas entre los } 60 \text { y } 65 \text { años-, los recursos con los que } \\
\text { cuenta la biblioteca para su desarrollo, y el tipo de evaluación } \\
\text { que se realiza }\end{array}$ \\
\hline $\begin{array}{l}\text { COMENTARIOS Y } \\
\text { OBSERVACIONES }\end{array}$ & $\begin{array}{l}\text { Es el apartado más flexible y abierto de la encuesta en donde se } \\
\text { invita a cada participante a comentar, reflexionar, sugerir, } \\
\text { resaltar, aclarar o compartir cualquier aspecto que pueda resultar } \\
\text { de relevancia para el análisis de los datos proporcionados en la } \\
\text { encuesta }\end{array}$ \\
\hline
\end{tabular}

\section{ANÁLISIS DE RESULTADOS}

Inicialmente, la encuesta se mantuvo activa hasta el 30 de noviembre de 2.014 y se cumplimentaron un total de 139 encuestas correspondientes a 89 bibliotecas; pero posteriormente, -tal y como hemos mencionado con anterioridad- y en vista del interés de otras bibliotecas por seguir participando, decidimos mantenerla abierta hasta marzo de 2.015. Así, a fecha de 13 de marzo de este año, se han 
presentado 178 encuestas correspondientes a 130 bibliotecas. Esto quiere decir, que hay bibliotecas que han cumplimentado más de una encuesta porque precisamente ofrecen o han ofrecido más de un servicio/recurso/actividad orientada a personas mayores o en la que, aún no estando pensada exclusivamente para ellos, su participación o implicación cumplían un papel fundamental. Evidentemente, estamos ante una muestra que puede resultar poco representativa cuantitativamente hablando- del conjunto de bibliotecas públicas españolas, pero es cierto que de las encuestas cumplimentadas hasta la fecha indicada es posible extraer unas primeras conclusiones e interpretaciones.

En primer lugar, y como ya hemos indicado, son 178 encuestas que corresponden a 130 bibliotecas. Las bibliotecas participantes han sido 18 Bibliotecas Públicas del Estado, 109 Bibliotecas Municipales (entre puntos de servicio o redes municipales de bibliotecas), y 3 Bibliotecas Regionales o Centrales de Comunidad Autónoma. Asimismo, ha llegado alguna encuesta procedente de otra tipología bibliotecaria: una encuesta de una biblioteca de hospital, otra de un centro coordinador de bibliobuses, y una procedente de la Biblioteca Nacional de Madrid ${ }^{5}$. Por áreas geográficas, las bibliotecas más participativas en este muestreo son las de la Región de Murcia y la Comunidad Foral de Navarra (24 y 22 encuestas cada una, respectivamente ${ }^{6}$ ), seguidas de Cataluña (-principalmente, bibliotecas de la provincia de Barcelona-, 21 encuestas) y Castilla y León (12 encuestas). En general, están representadas todas las Comunidades Autónomas, excepto Ceuta y Melilla de las que no ha llegado información alguna sobre sus bibliotecas, lo que podría dar a entender que no realizan o han realizado nunca alguna actividad o proyectado algún servicio orientado para el colectivo de personas mayores.

Una inmensa mayoría de las encuestas que han llegado, han respondido afirmativamente que ofrecen o han ofrecido alguna actividad o servicio para personas mayores. Concretamente, Un $90 \%$ de las bibliotecas que han respondido a la encuesta confirman este particular, mientras que un $10 \%$ indican que nunca han organizado actividad o servicio alguno con el colectivo de mayores en mente. Una primera lectura de estos datos es que la casi la totalidad de las bibliotecas que han participado en nuestra encuesta ofrecen servicios o actividades dirigidas al colectivo de personas mayores.

${ }^{5}$ La encuesta procedente sobre la Biblioteca Nacional de Madrid fue cumplimentada por una persona que se identificaba como voluntario de esta institución

${ }^{6}$ En el caso de la Comunidad Autonóma de Murcia, pensamos que es la región española más representada por el hecho de haber difundido la existencia de esta encuesta a través de la lista de distribución BiblioMun (Red de Bibliotecas Públicas Municipales de la Región de Murcia) http://www.redbibliotecas.carm.es/RedBibliotecas/faces/generaPagina.jsp;jsessionid=84F76014 A1E2EBE8BF9188F8C0A009F9.nd1forocul?idioma=1\&pagina=321 
Tabla 2. Número de encuestas cumplimentadas, según Comunidad Autónoma

\begin{tabular}{|c|c|}
\hline Comunidad Autónoma & Número de encuestas \\
\hline Murcia & 24 \\
\hline Navarra & 22 \\
\hline Cataluña & 21 \\
\hline Castilla y León & 12 \\
\hline Andalucía & 11 \\
\hline Canarias & 9 \\
\hline Asturias & 9 \\
\hline Valencia & 9 \\
\hline Aragón & 9 \\
\hline Castilla-La Mancha & 9 \\
\hline La Rioja & 7 \\
\hline Madrid & 7 \\
\hline Extremadura & 6 \\
\hline País Vasco & 6 \\
\hline Galicia & 6 \\
\hline Cantabria & 6 \\
\hline Baleares & 5 \\
\hline Ceuta y Melilla & 0 \\
\hline & \\
\hline
\end{tabular}

De las 118 bibliotecas que ofrecen algún servicio, recurso o actividad para mayores, un 74,5\% (88 bibliotecas) señala que es uno de los colectivos con los que trabajan habitualmente, siendo el 23,5\% restante que lo hacen más puntualmente. Este dato indica, al menos entre las bibliotecas participantes en la encuesta, que existe un interés cada vez mayor en ampliar la oferta bibliotecaria a estas personas.

Las bibliotecas que respondieron no haber trabajado con el colectivo de mayores (el $10 \%$ de las bibliotecas que han participado en la encuesta) argumentaron como razones más relevantes que nunca se lo habían planteado (4 bibliotecas), que no sabían qué actividad o servicio ofrecerles (3), ni cómo plantearles participar en las actividades de la biblioteca (3). Otras razones aducidas fueron que resulta difícil acceder a este colectivo por encontrarse muy disperso en el municipio (1), que no suelen visitar nunca la biblioteca (1), o que no tienen los medios necesarios para ofrecer actividades o servicios de su interés (1).

En cuanto a los usos que hacen de las bibliotecas, hay que mencionar que esta pregunta era abierta y se dejaba que cada biblioteca indicase o expresase que servicio o servicios son utilizados por sus usuarios mayores. Así, el préstamo de libros se menciona en 102 ocasiones, y la lectura de prensa y revistas en 94 constituyéndose pues en los usos mayoritarios que hacen las personas mayores de las bibliotecas. Más de lejos se menciona otros usos como: la participación en talleres de alfabetización digital en 31 ocasiones, acceso a Internet en 27 ocasiones, la asistencia a actividades culturales (como conferencias, charlas, exposiciones, 
presentaciones de libros, etc.) en 26 ocasiones, la participación en clubes de lectura en 19 ocasiones, por citar algunos de los más aludidos en las encuestas.

Tabla 3. Uso de servicios y número de menciones en la encuesta

\begin{tabular}{|l|l|}
\hline Servicio/s mencionado/s & Número de menciones \\
\hline Préstamo de libros & 102 \\
\hline Lectura de prensa y revistas & 94 \\
\hline Alfabetización digital & 31 \\
\hline Acceso a Internet & 27 \\
\hline Asistencia a actividades culturales & 26 \\
\hline Participación en clubes de lectura & 19 \\
\hline Lectura en sala & 18 \\
\hline Préstamo de material audiovisual & 17 \\
\hline
\end{tabular}

A la pregunta de si la biblioteca cuenta con un espacio o área específicamente pensada para los mayores, la práctica totalidad responde negativamente justificando en muchas ocasiones tal respuesta. Según las respuestas proporcionadas, el objetivo sería conseguir su integración con el resto de visitantes a la biblioteca.

En cuanto a las condiciones de accesibilidad que presentan las bibliotecas encuestadas y su adaptación a las características físicas de las personas mayores, prácticamente un $50 \%$ afirma que su biblioteca no reúne las condiciones de accesibilidad necesarias. No obstante, alrededor de un $70 \%$ de estas bibliotecas prepara adoptar medidas en este sentido a corto plazo. Entre las bibliotecas que sí presentan medidas de accesibilidad, los sistemas que se mencionan para facilitar esta accesibilidad son fundamentalmente rampas de acceso, ascensores, puertas anchas, aseos adaptados, acceso directo desde la calle, o pasamanos en las escaleras cuando no existe ascensor para subir o bajar de una planta a otra. Esta cuestión adquiere una gran relevancia ya que determinadas discapacidades físicas de los mayores suelen estar asociadas al factor edad y por tanto un requisito fundamental para acercar las bibliotecas a los mayores, y viceversa, es que cuenten con las condiciones de accesibilidad necesarias (CEAPAT-IMSERSO, 2011).

En nuestra encuesta también queríamos averiguar la existencia o no de secciones o servicios específicos para personas mayores en las bibliotecas públicas españolas. En líneas generales, no proliferan en las bibliotecas participantes en la encuesta la existencia de secciones o servicios específicos para usuarios mayores, aunque también es cierto que en aquellas que responden afirmativamente (un $37,2 \%$ ) se mencionan secciones o servicios como los siguientes: secciones de libros escritos en grandes caracteres, centros de interés 
con información y recursos sobre temas de interés para estas personas, colecciones de audiolibros, o las secciones de lectura fácil que últimamente tienden a incorporarse a la oferta de servicios bibliotecarios (Mayol 2012).

Figura 3. Centro de interés “Personas mayores” de Biblioteca Horta Can Mayor de Barcelona

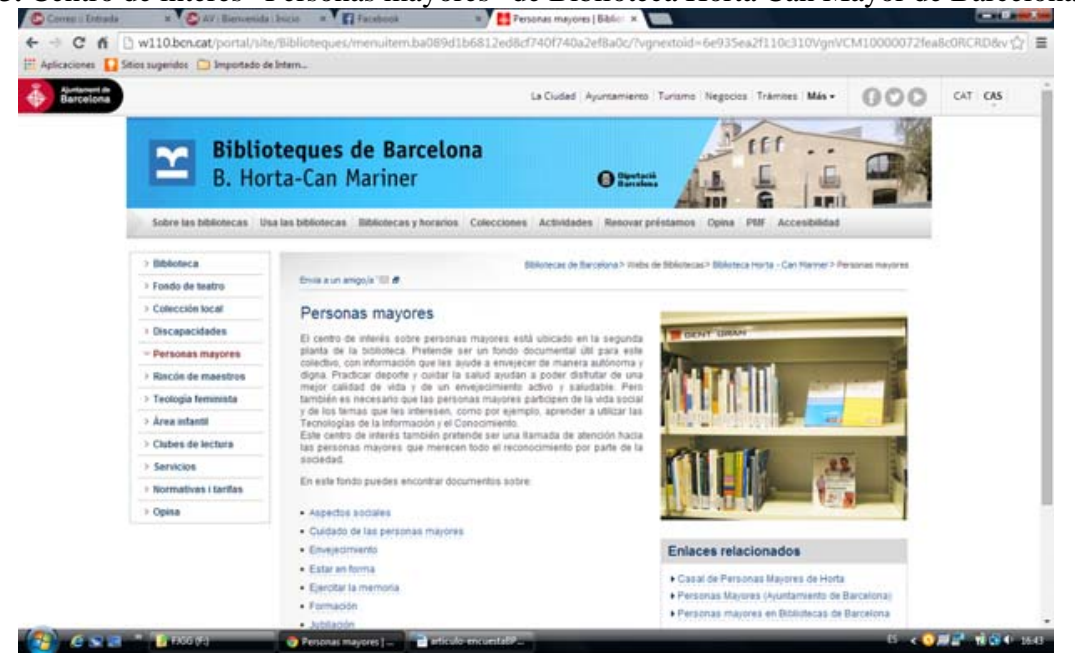

La encuesta también se fija en la oferta de servicios y recursos digitales que pueden ofrecer estas bibliotecas a este colectivo. Nos interesa conocer la existencia o no de un apartado o sección específica dedicada a las personas mayores en la sede web de la biblioteca. Lo cierto es que entre las bibliotecas que cuentan con sede web -que son la inmensa mayoría de las bibliotecas que responden a la encuesta-, son más bien pocas (en torno a un $14,6 \%$ de las bibliotecas participantes en la encuesta) las que dicen ofrecer contenidos o recursos digitales de interés o pensados específicamente en las personas mayores. De estas bibliotecas que responden afirmativamente, podemos establecer la siguiente tipología de servicios, secciones o contenidos: página web temática, selección de recursos digitales ordenados temática o alfabéticamente, y guías temáticas o bibliográficas. Estos datos ponen de manifiesto una carencia de servicio en las bibliotecas públicas españolas en este sentido, ya que no se considera al colectivo de los mayores como un usuario potencial de sus servicios o recursos web y, por tanto, se abre aquí un campo de trabajo para el futuro a corto o medio plazo para las bibliotecas públicas españolas (García y Díaz, 2004). El caso de la sección "Gent Gran" que ofrece en su sede web la Red de Bibliotecas Municipales de Barcelona http://www.bcn.cat/biblioteques puede ser un claro ejemplo a seguir en este sentido. 
Figura 4. Sección web “Gent gran” de Biblioteques de Barcelona

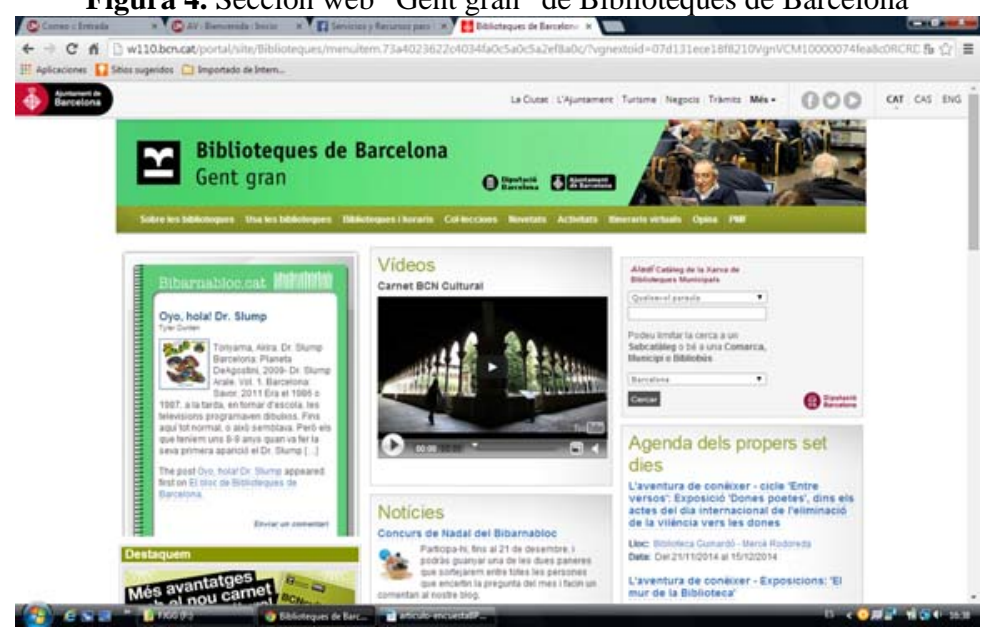

A continuación, la encuesta centra su atención en el servicio, proyecto, sección o actividad concreta sobre el que la biblioteca participante informa o desarrolla en detalle. Hemos tenido en cuenta tanto el hecho de que sea una acción o proyecto pensado exclusivamente para los mayores como aquellas otras en las que, sin ser los destinatarios principales de la actividad o servicio, sí son parte importante del desarrollo del mismo. Encontramos una gran variedad tanto en cuanto a su finalidad, en cuanto a su dinámica, y también en cuanto a su denominación. Así, nos podemos encontrar con títulos tan sugerentes como "Labrantes de la palabra", "Mi primera vez en San Javier", "Taller de memoria”, "Taller de smartphone”, "Los mayores también cuentan” o “Te presto mis ojos”, por citar unos cuantos.

Figura 5. Taller de smartphone para mayores de Bibliotecas Municipales de Madrid

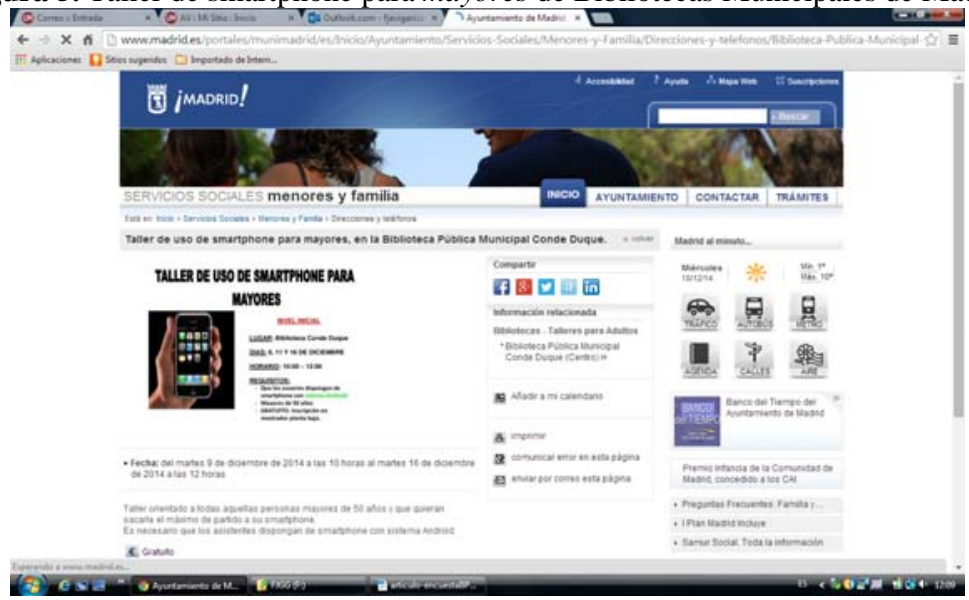


Son actividades, recursos, colecciones, servicios o proyectos que giran en torno al fomento de la lectura, la extensión bibliotecaria, la formación y el aprendizaje, el fomento de las relaciones intergeneracionales, la recuperación de la memoria histórico-local, la información a medida, y el voluntariado. Así, nos podemos encontrar con talleres de alfabetización digital, servicios bibliotecarios a domicilio para mayores impedidos, talleres de escritura o de lectura tanto presenciales como incluso virtuales (Moreno Mulas, 2006), encuentros intergeneracionales, programa de visitas guiadas a la biblioteca con residencias de ancianos, sesiones de abuelos cuentacuentos, secciones y centros de interés para mayores, talleres para ejercitar la memoria, encuentros para la recuperación de la memoria histórica local, etc. Los objetivos que persiguen estas actuaciones son diferentes: el fomento de la lectura, la inclusión social y digital, fomentar las relaciones intergeneracionales, la recogida de testimonios orales, entre otros muchos.

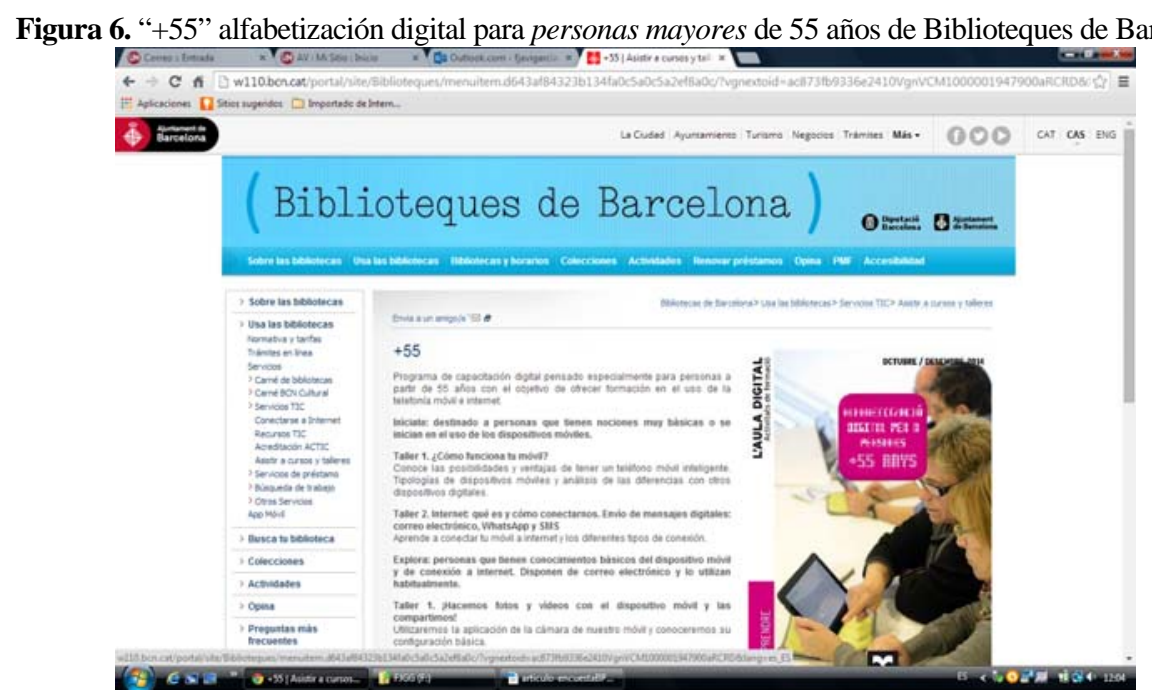

Son, en su mayoría, actuaciones bibliotecarias en curso o proyectos abiertos, que viene realizándose desde hace tiempo -una media de entre tres o cuatro años, aunque nos encontramos con casos de servicios que llevan funcionando una década como es el proyecto "Labrantes de la palabra" de la Biblioteca Municipal de Arucas (Gran Canaria)-, sin fecha de "caducidad" mientras exista demanda, que en algunos casos dependen de apoyo económico o institucional (como el caso del programa de voluntariado cultural "Biblioteca Solidaria" coordinado por la Biblioteca Pública del Estado de Cuenca, y disponible en las bibliotecas públicas de Castilla-La Mancha) frente a una minoría de servicios o actividades que se han venido realizando tiempo atrás y que posteriormente no se prosiguieron 
celebrando por diferentes razones. Otras bibliotecas indican que, aunque no han trabajado con personas mayores, sí tienen en mente la puesta en marcha de alguna actividad o servicio a corto plazo, especialmente -así nos los indican algunas bibliotecas participantes en la encuesta- a raíz de este estudio y del hecho de que se percibe en el entorno bibliotecario un mayor interés en este colectivo de usuarios.

Figura 7. "Biblioteca solidaria”, voluntariado cultural en Bibliotecas de Castilla-La Mancha

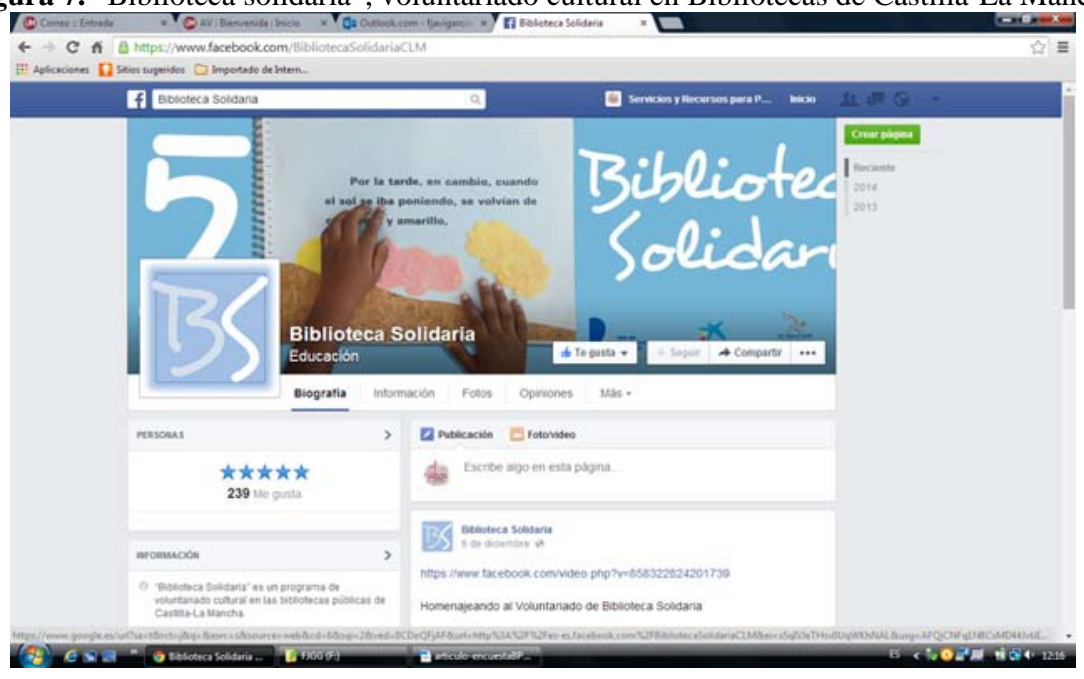

Otra cuestión por la que preguntábamos era por los destinatarios concretos de la actividad, servicio o proyecto, en el sentido de conocer si se ofertaba para la comunidad de mayores en general o, si por el contrario, estaba enfocada hacia algún tramo de edad concreto. La inmensa mayoría de las respuestas apuntaron hacia "personas mayores en general", aunque también recibimos respuestas más minoritarias del tipo "para mayores de 55 años", "tercera edad”, “jubilados”, o “cualquier persona mayor con dificultades visuales y/o de comprensión”. En otros casos, también minoritarios, algunas bibliotecas apuntaban al hecho de que la actividad o servicio estaba ideado para intervenir tanto personas mayores como niños especialmente cuando se referían a la celebración de actividades o encuentros intergeneracionales. Estas respuestas ponen en evidencian que, por el momento, los servicios y recursos ofrecidos para personas mayores no contemplan la segmentación por tramos de edad (por ejemplo, para mayores de 55 años y hasta 64 años, para mayores de 65 años, etc.), para ajustar sus características físicas, motrices, intelectuales o psicológicas a las peculiaridades del servicio o proyecto bibliotecario.

En cuanto a los medios técnicos, humanos, materiales o tecnológicos necesarios para la puesta en marcha de los servicios o recursos bibliotecarios reseñados para personas mayores en la encuesta, encontramos respuestas muy diferentes que tienen 
mucho que ver con la dinámica propia de la actividad, servicio o recurso que ofrece la biblioteca en cuestión. Así, por ejemplo, en los servicios bibliotecarios "a domicilio" en los que la biblioteca pone en marcha un servicio de préstamo de documentos o de acercamiento de la lectura a personas mayores impedidas en su hogar o en una residencia de ancianos, es necesario medio de transporte (alguna biblioteca ha señalado que es el propio personal bibliotecario quien se desplaza en su vehículo y que el ayuntamiento de quien depende dicha biblioteca le abona gastos de kilometraje), y personal para desplazarse hasta los lugares de residencia de los destinatarios del servicio (pudiendo ser tanto el personal bibliotecario, o bien voluntarios colaboradores, familiares del mayor impedido que colaboran con la biblioteca, monitores, estudiantes en periodo de prácticas, o incluso personal especializado externo a la biblioteca de alguna institución social que colabora con la biblioteca en la prestación del servicio). En este apartado, varias bibliotecas han comentado que la existencia de colaboradores o voluntarios en la prestación especialmente de los llamados "servicios a domicilio" es imprescindible y que, sin estas personas, difícilmente podrían ofrecerlo como hasta ahora. Una primera lectura de esto que comentamos indica que los medios económicos o materiales con los que cuentan muchas biblioteca son limitados -algo que se acentúa en el contexto económico actualy que necesitan, pues, de la implicación de parte de su comunidad para poder prestarlos, muchas veces de la acción cooperativa con otras bibliotecas o instituciones socio-culturales, y otras veces echar mano de la imaginación y la inventiva para sacar adelante los servicios que ofrecen a este colectivo de ciudadanos.

Figura 8. Servicio “TeleBiblioteca” que ofrecen las bibliotecas públicas de la Comunidad de Madrid

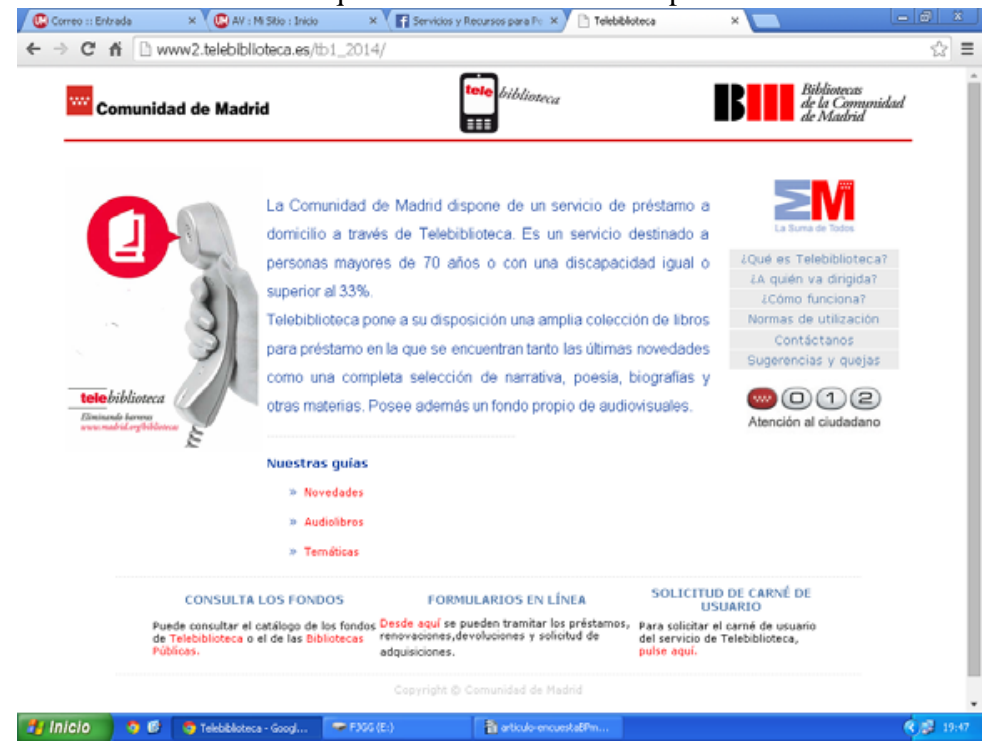


En los casos en los que el servicio o actividad se ofrece en las instalaciones de la biblioteca, las necesidades incluyen además del personal necesario para su desarrollo (que puede coincidir con la misma tipología que hemos comentado en el caso de servicios a domicilio), la infraestructura y logística precisa que puede ir desde un espacio físico concreto para la llevar a cabo una acción formativa, recursos tecnológicos y/o audiovisuales, espacios o salas para debatir o compartir conocimientos y vivencias, etc. Algunas bibliotecas han declarado contar con espacios físicos adecuados para ofrecer las actividades o servicios orientados para personas mayores, pero también otras bibliotecas han reconocido que el hecho de no contar con las instalaciones o espacios físicos precisos se convierte en una dificultad añadida, teniendo muchas veces que optar por ofrecer sus servicios en lugares distintos a la propia biblioteca (en una residencia de ancianos, en un centro de día de mayores, en un centro cívico,...).

La encuesta también considera la evaluación realizada por las bibliotecas para conocer el grado de cumplimiento de los objetivos planteados de antemano. De las bibliotecas que ofrecen alguna actividad o servicio para mayores, aproximadamente un 41,5\% reconoce realizar tareas de evaluación, mientras que el resto asegura no realizar evaluación alguna. Entre las bibliotecas que afirman evaluar sus actividades y servicios con mayores, casi un 70\% utiliza encuestas de satisfacción que es el medio que prevalece sobre otras técnicas o sistemas de evaluación. Muy lejos de las encuestas de satisfacción aparecen otros procedimientos como las estadísticas de préstamos o de uso de servicio, el número de participantes a las actividades programadas, el número de voluntarios mayores participantes, la observación, las estadísticas de visita y accesos a la sección web, o la revisión periódica de las colecciones o secciones específicas para valorar el mayor o menor uso de las mismas.

Figura 9. Taller de alfabetización digital para mayores con discapacidad auditiva en la Biblioteca Regional de La Rioja

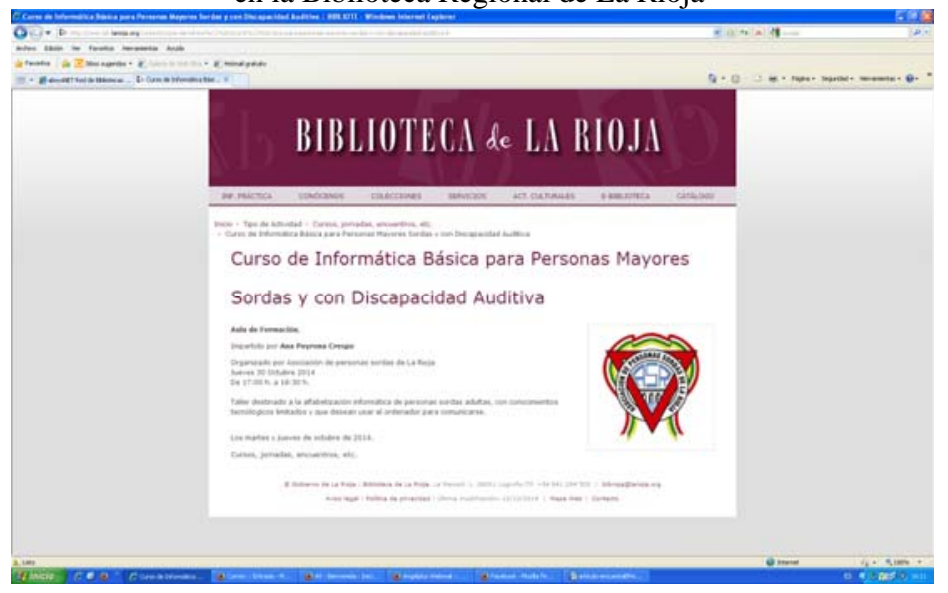


Como último apartado, la encuesta presentaba una sección para que cada biblioteca participante pudiera exponer cualquier tipo de observación o comentario sobre su participación en la encuesta. En este apartado final, las bibliotecas aprovechan para concretar aspectos de sus actividades o servicios con los mayores, para aportar detalles específicos sobre la relación que mantienen con este colectivo, algunas bibliotecas incluso aprovechan este espacio para justificar por qué trabajan o no trabajan habitualmente con personas mayores, en definitiva para aportar información complementaria a la que van incorporando en la encuesta. Algunos comentarios que van dejando las bibliotecas son realmente llamativos como los que reproducimos a continuación: "Mis mejores usuarios son personas mayores, quizás porque tienen más tiempo para leer o igual mayores inquietudes (me sorprende tener usuarios de 60 años y muy pocos de 25 por ejemplo). Mi usuario favorito es un señor de 80 años que apenas ve por culpa de cataratas y aún así se lleva libros en préstamo, es algo admirable”. En este misma línea se pronuncia la biblioteca Municipal de Moratalaz (Madrid) cuando expone que "(...) Es especialmente gratificante que los usuarios mayores expresen su agradecimiento por el servicio prestado, o su satisfacción por la lectura de una determinada obra proporcionada por la biblioteca". Finalmente, la diversidad de los usos que realizan de las bibliotecas queda manifiesta con lo que nos cuentan desde la Biblioteca Municipal de Ceutí (Murcia) "La hemeroteca para la lectura de los periódicos diarios. Y acuden a los talleres de lectura con mucha frecuencia. Una minoría comienza a utilizar sus propios portátiles aprovechando la wifi. Y si proponemos formación en TIC para mayores, también acuden sobre todo si lo hacemos a través de colectivos específicos de mayores. Otro tipo de mayores, como los abuelos, acuden con los nietos a la sala infantil. En los servicios de Préstamo se llevan más libros que audiovisuales y en Internet su consulta es minoritaria frente a personas más jóvenes. Los mayores también acuden a actividades culturales".

Figura 10. "Labrantes de la palabra” de la Biblioteca Municipal de Arucas (Gran Canaria)

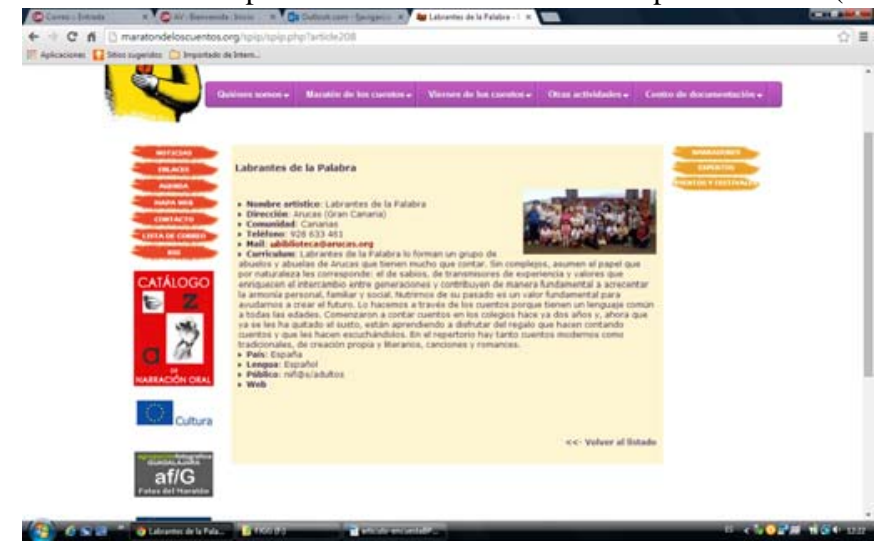

Revista General de Información y Documentación 


\section{CONCLUSIONES}

En líneas generales, y a tenor de los datos ofrecidos por las bibliotecas participantes en la encuesta, podemos extraer como primera conclusión que se detecta un interés creciente por parte de las bibliotecas españolas en este colectivo. Aunque algunas bibliotecas han visto reconocido su esfuerzo y dedicación con este colectivo de usuarios, como es el caso de las Bibliotecas Municipales de Barcelona $^{7}$ o el más reciente de la Biblioteca Municipal de Castropol (Asturias), es cierto que aún queda mucho camino por recorrer. No debemos olvidar algunas de las razones que las bibliotecas que han respondido que no trabajan con personas mayores porque constituyen claros desafíos que tienen estas instituciones por delante.

Influye también el contexto de restricciones presupuestarias en el que se desenvuelven los servicios públicos en general, y las bibliotecas públicas españolas en particular, ya que afectan directamente en la prestación del servicio, en la calidad del mismo, y en la consecución de los objetivos y fines previstos. Es un entorno en cierto modo amenazador para las bibliotecas, pero al mismo tiempo sugerente y abierto a la experimentación y la innovación (García Gómez, 2013)

Las principales conclusiones que podemos extraer de los resultados de esta encuesta son las siguientes:

${ }^{7}$ La Red de Bibliotecas Municipales de la ciudad de Barcelona se está convirtiendo en un referente bibliotecario a nivel estatal, tanto por sus métodos de trabajo, sus proyectos desarrollados, su enfoque hacia el usuario, o el hecho de ser considerado como el mejor servicio municipal según los propios barceloneses. También destacan estas bibliotecas por su carácter proactivo con el colectivo de personas mayores. En su Memoria de 2.012 mencionan la realización de un estudio sobre las actitudes y expectativas de los mayores con respecto al servicio bibliotecario ofrecido (p.75). El objetivo del estudio era conocer las causas por las que este colectivo no hacía un uso mayor de las bibliotecas http://w110.bcn.cat/Biblioteques/Continguts/Documents/BIBLIOTEQUES\%20DE\%20BARCE LONA\%20-\%20MEM\%C3\%92RIA\%202012.pdf Posteriormente, en 2.014, se crea un grupo de trabajo transversal en colaboración con otros agentes sociales de la comunidad para establecer sinergias que permita a las Bibliotecas Municipales de Barcelona afianzar su labor con el colectivo de mayores

${ }^{8}$ La Biblioteca de Castropol, con su proyecto "Facilitando lectura, integrando lectores" dirigido a personas mayores y a personas con movilidad reducida, obtuvo el accésit en el Premio "Biblioteca Pública y Compromiso Social” con convocado por la Fundación Biblioteca Social, una institución sin ánimo de lucro constituida en 2014, cuya misión consiste en contribuir a compensar los desequilibrios sociales existentes en España mediante la colaboración con las bibliotecas públicas en proyectos que llevan a cabo dirigidos a los sectores más vulnerables de la sociedad http://fundacionbibliotecasocial.org/es/premio-2015-bibliotecapublica-y-compromiso-social/ 
Los usos que mayoritariamente hacen los mayores de las bibliotecas están relacionados con la lectura de libros, revistas y prensa. El servicio de préstamo de libros es el servicio más utilizado, seguido de la lectura de prensa y revistas. Más lejos quedan el uso de otros servicios como el de acceso a Internet, la asistencia a actividades culturales (charlas, conferencias, audiciones, presentaciones de libros, etc.) o sacar en préstamo material audiovisual. Otros servicios utilizados por los mayores, pero en menores proporciones, son la participación en talleres o clubes de lectura, la lectura y consulta en sala en general, y la participación en talleres formativos relacionados con el uso de las tecnologías de la información

$>$ La integración con el resto de usuarios y tramos de edades es una de las razones más importantes que profesan las bibliotecas al justificar el hecho de que no existan espacios o áreas de servicio para uso exclusivo de los mayores

$>$ La accesibilidad es una tarea pendiente en el colectivo de bibliotecas participantes en la encuesta. Por suerte, muchas de las bibliotecas que reconocen no contar con las condiciones de accesibilidad necesarias, tienen como uno de sus próximos objetivos dotar de medidas de accesibilidad que hagan más fácil de utilizar las bibliotecas a todas aquellas personas que presentan algún tipo de discapacidad y, por ende, a las personas de edad avanzada

$>\mathrm{Al}$ igual que no existen áreas o servicios específicos en las instalaciones bibliotecarias, tampoco está muy extendida la existencia de secciones o colecciones diseñadas para el uso de personas mayores. No obstante, algunas bibliotecas sí mencionan contar precisamente con pequeñas secciones o colecciones de documentos cuyos destinatarios ideales sería el colectivo de personas mayores. Se citan en este sentido, los centros de interés con documentos que tratan temas de interés para personas mayores, las colecciones de libros escritos en grandes caracteres, los audiolibros, o las secciones con materiales de lectura fácil que poco a poco van encontrando sitio en la oferta de recursos de muchas bibliotecas

$>$ La presencia de servicio y recursos para personas mayores en los sitios web de las bibliotecas participantes en la encuesta es uno de las carencias más relevantes que se mencionan. De hecho, son más bien pocas las que reconocen ofrecer contenidos adecuados y/o de interés para mayores en sus espacios web

$>$ En cuanto a los proyectos de trabajo reseñados con y/o para mayores, hay que decir que encontramos una gran variedad de actividades y servicios lo que debe ser valorado muy positivamente. En este sentido, hay que resaltar el hecho de que la oferta bibliotecaria existente para el colectivo de mayores es muy rica y variada abarcando el fomento de la lectura, la 
inclusión social y digital, la generación de relaciones intergeneracionales, la recuperación de la memoria histórico-local, entre otros. De hecho, podemos clasificar los servicios y recursos bibliotecarios para personas mayores en seis grandes categorías: fomento de la lectura; extensión bibliotecaria; formación y aprendizaje; fomento de relaciones intergeneracionales; recuperación de la memoria del lugar; información y recursos a medida; y participación mediante voluntariado

$>$ La inmensa mayoría de proyectos de trabajo informados en la encuesta se trata de proyectos en curso, algunos de ellos incluso con una dilatada trayectoria en el tiempo, y que tienen previstos seguir prestándose debido a su buena aceptación y los buenos resultados cosechados. Algunos de estos proyectos precisan de determinados medios económicos, humanos y de apoyo institucional para continuar ofreciéndose, tal y como han puesto de manifiesto las bibliotecas implicadas en los mismos

$>$ La planificación de las prestaciones bibliotecarias para mayores no contemplan la segmentación por edades de este colectivo, sino que en principio son ofrecidas para personas mayores en general, mayores de 55 o 65 años según los casos, sin que suponga un inconveniente o un requisito indispensable contar con una determinada edad

$>$ Las bibliotecas participantes en la encuesta manifiestan no llevar a cabo siempre tareas de evaluación de las actividades y servicios ofrecidos a los mayores. Aquellas que sí lo realizan, declaran utilizar como sistema de evaluación mayoritario las encuestas de satisfacción. Otros mecanismos utilizados son las estadísticas de uso de los servicios tanto presenciales como virtuales o la revisión periódica de las colecciones o secciones específicas para controlar el grado de uso de las mismas

Somos conscientes de que la muestra de bibliotecas participantes en esta encuesta resulta cuantitativamente poco representativa del conjunto de bibliotecas públicas españolas, aunque consideramos que los datos extraídos ponen de relieve la necesidad de seguir investigando sobre este particular al tiempo que sirve de punto de partida que puede dar lugar a muchas y variadas investigaciones en este campo de estudio aportando información que puede ser muy valiosa para diseñar acciones bibliotecarias para este colectivo en un futuro cercano, ya que su presencia e importancia en la sociedad española irá en crecimiento.

Para concluir, queremos dejar constancia de que las personas mayores son una fuente de conocimientos y sabiduría que, de alguna manera, no se está sabiendo aprovechar convenientemente, dado el valor añadido que pueden aportar a las bibliotecas en tanto que servicio público y de recursos para su comunidad (Martínez, 2013). Los mayores pueden encontrar en la oferta de servicios y actividades bibliotecarias un medio de evasión y entretenimiento, una excusa para sentirse vivos y útiles, beneficiándose de los efectos positivos de la lectura, de las 
manifestaciones culturales, del acceso a todo tipo de información, o de la adquisición de habilidades informacionales y digitales que ofrece la biblioteca, y beneficiando a su comunidad al transmitir y compartir su experiencia de vida. Por todo ello, somos partidarios de facilitar al máximo la implicación y participación de los mayores en las bibliotecas de su comunidad. Pongamos una biblioteca en la vida de las personas mayores. Todos saldremos ganando.

\section{BIBLIOGRAFÍA}

ARES SEIJO, Ivana (2014). “Gent gran a la biblioteca: més enllá de la lectura del diari” [En línea]. ITEM, nº36 (2004), p. 41-57. Disponible en: www.raco.cat /index.php/Item/article/view/22613/22447 [consulta: 8/2/2015]

CEAPAT-IMSERSO (2011). Bibliotecas accesibles para todos: pautas para acercar las bibliotecas a las personas com discapacidad y a las personas mayores. [En línea]. Madrid: Ministerio de Sanidad, Política Social e Igualdad. Disponible en: travesia.mcu.es/portalnb/jspui/handle/10421/5761 [consulta: 8/2/2015]

GARCÍA GIMÉNEZ, Daniel (2010). "Redes sociales: posibilidades de facebook para las bibliotecas públicas” [En línea]. BiD, n²4 (2010). Disponible en: bid.ub.edu/24/garcia2.htm [consulta: 8/2/2015]

GARCÍA GÓMEZ, Fco. Javier (2014). "Cuando la edad no es una excusa: recursos y servicios bibliotecarios para personas mayores”. Actas VII Congreso Nacional de Bibliotecas Públicas (Badajoz, 12-14 de noviembre de 2014), [en prensa]

- (2013). "Innovación en la biblioteca pública española: dónde estamos y hacia dónde vamos”. Revista General de Información y Documentación, vol.23, n¹, pp. 133-150

- (2008). "Integrando a los mayores en la sociedad digital: propuestas desde la biblioteca pública”. En: Actas del IV Congreso Nacional de Bibliotecas Públicas (A Coruña), pp. 631-642.

GARCÍA GÓMEZ, Fco. Javier y DÍAZ GRAU, Antonio (2004). "Servicios bibliotecarios para la tercera edad en entornos web: experiencias desarrolladas en bibliotecas públicas”, en El Profesional de la Información, vol.13, nº (2004), pp. 272-280.

- Antonio (2001). "Los usuarios de la tercera edad en la biblioteca pública: nuevo reto para la formación de usuarios”. Educación y Biblioteca, nº120, pp. 26-28.

MARTÍNEZ, Fuensanta (2013). "La importancia de los usuarios mayores” [En línea]. BiblogTecarios. Disponible en: http://www.biblogtecarios.es/ fuensantamartinez/la-importancia-de-los-usuarios-mayores/. [consulta: 16/1/2013] 
MAYOL, Carme y SALVADOR, Eugenia (2012). "La Lectura Fácil: accions més destacables del bienni 2010-2011”. En: Anuari de l'Observatori de biblioteques, Llibres i Lectura, vol.2, pp. 98-105.

MORENO MULAS, M ${ }^{a}$ Antonia (2006). "Bibliotecas públicas: no tengo edad para esto, una experiencia de formación en las TIC”, en Educación y Biblioteca, $\mathrm{n}^{\circ} 156$ (2006), pp. 97-100.

OVALLE PERANDONE, M ${ }^{\mathrm{a}}$ Antonia y GUARDADO GARCÍA, Sonia (2013). "Los servicios 1.0 y 2.0 en las bibliotecas públicas en línea de los municipios de Bizkaia (euskadi)” [En línea]. BiD, $\mathrm{n}^{0} 31$. Disponible en: bid.ub.edu/es/31/ovalle2.htm. [consulta: 16/1/2013]

PÉREZ DÍAZ, Julio (2010). "El envejecimiento de la población española". Investigación y Ciencia, noviembre de 2010, pp. 35-42

SALONI MARIMÓN, Guadalupe (2008). "Biblioteca y mayores: la ola perfecta de no-viejos se acerca a la playa de la biblioteca pública” [En línea] BiD, nº21. Disponible en: http://bid.ub.edu/21/salon2.htm. [consulta: 16/1/2013]

UROZ OLIVARES, Jorge (2010). "La llamada crisis del modelo de estado de bienestar: reestructuración y alternativas” [En línea]. Miscelánea Comillas, vol.68, n¹32, pp. 299-311

\section{Anexo: la encuesta}

Biblioteca:

Fecha de cumplimentación de la encuesta:

Persona que participa en la encuesta:

Cargo en la biblioteca:

\section{USO DE LA BIBLIOTECA}

1. ¿Acuden a la biblioteca personas mayores?

Si su respuesta es positiva, indique:

2. ¿Qué servicios de la biblioteca suelen utilizar habitualmente? (sea lo más específico posible)

\section{ESPACIOS Y AREAS FISICAS DE LA BIBLIOTECA}

3. ¿Cuenta la biblioteca con un espacio o area especifica para personas mayores?

4. Si su respuesta es positiva, describa brevemente dicha area o espacio fisico

5. ¿Considera que su biblioteca reune condiciones de accesibilidad para personas mayores con dificultades de desplazamiento?

6. Si su respuesta es positiva, indique las medidas de accesibilidad disponibles 
7. Si su respuesta es negativa, indique si tiene previsto adoptar en breve medidas de accesibilidad

\section{SECCIONES Y COLECCIONES DE LA BIBLIOTECA}

8. ¿Cuenta la biblioteca con una sección especifica dedicada a información o recursos de interes para personas mayores?

9. Si su respuesta es positiva, describa brevemente dicha sección

\section{SEDE WEB DE LA BIBLIOTECA}

10. ¿Cuenta la biblioteca con un apartado de su sede web dedicado específicamente a suministrar información de interés para personas mayores?

\section{SERVICIOS Y ACTIVIDADES \\ NOMBRE DEL SERVICIO/RECURSO/ACTIVIDAD/PROYECTO:}

11. ¿Es un servicio/recurso/actividad/proyecto en curso?

12. Si su respuesta es negativa, indique el año o periodo durante el que se ofreció/desarrolló ese servicio/recurso/actividad/proyecto

13. Si su respuesta es negativa, indique si tiene previsto la biblioteca ofertar algún servicio/recurso/actividad/proyecto para personas mayores a corto/medio plazo

14. Si su respuesta es positiva, indique el tiempo previsto que se mantendrá ese servicio/recurso/actividad/proyecto operativo

\section{CARACTERÍSTICAS DEL}

\section{SERVICIO/RECURSO/ACTIVIDAD/PROYECTO}

15. (Haga una breve descripción del mismo)

\section{OBJETIVOS DEL SERVICIO/RECURSO/ACTIVIDAD/PROYECTO}

16. (Indique el objetivo general y/o objetivos específicos que se pretenden conseguir)

\section{DESTINATARIOS DEL SERVICIO/RECURSO/ACTIVIDAD/PROYECTO}

17. (Especifique si es para los mayores en general, si es a partir de una determinada edad -por ejemplo, a partir de 75 años-, si es para mayores y niños, etc.)

18. Medios humanos, materiales y de otro tipo necesarios para el desarrollo del servicio/recurso/actividad/proyecto (si fueran necesarios)

19. ¿Realiza la biblioteca algún tipo de evaluación de los servicios/recursos/actividades/proyectos desarrollados para personas mayores?

20. Si su respuesta es positiva, indique el tipo de evaluación realizada

21. Observaciones y comentarios 